\title{
CERTAIN CLASSES OF MEROMORPHIC $p$-VALENT FUNCTIONS WITH POSITIVE COEFFICIENTS
}

\author{
MOA'ATH NASSER AND MASLINA DARUS
}

\begin{abstract}
We introduce the new class $L(\alpha, \beta, \lambda, p)$ of meromorphic p-valent functions. The aim of the paper is to obtain coefficient inequalities, growth and distortion, radii of convexity and starlikeness and the convex linear combinations for the class $L(\alpha, \beta, \lambda, p)$.
\end{abstract}

\section{Introduction}

Let $\Sigma_{p}$ denotes the class of the form

$$
f(z)=\frac{1}{z^{p}}+\sum_{n=1} a_{p+n-1} z^{p+n-1}
$$

which are analytic and p-valent in a punctured disc $D=\{z: 0<|z|<1\}$. Further let $\Sigma_{p}^{*}(\alpha)$ be the class of $\Sigma_{p}$ consisting of function $f$ which satisfies the inequality

$$
\operatorname{Re}\left(-\frac{z f^{\prime}(z)}{f(z)}\right)>\alpha, \quad(0 \leq \alpha<p) .
$$

And let $\Sigma_{p}^{C}(\alpha)$ be the class of $\Sigma_{p}$ consisting of function $f$ which satisfies the inequality

$$
\operatorname{Re}\left(-1-\frac{z f^{\prime \prime}(z)}{f^{\prime}(z)}\right)>\alpha, \quad(0 \leq \alpha<p) .
$$

Clearly, we have

$$
f \in \Sigma_{p}^{C}(\alpha) \Longleftrightarrow z f^{\prime}(z) \in \Sigma_{p}^{*}(\alpha),(0 \leq \alpha<p, p \in N) .
$$

This condition is obviously analogous to the well-known Alexander equivalent (see for details [1]). Many important properties and characteristics of various interesting subclasses of the class $\Sigma_{p}$ of meromorphic p-valent functions, including the classes $\Sigma_{p}^{*}(\alpha)$

Received April 13, 2005.

2000 Mathematics Subject Classification. 30C45.

Key words and phrases. Meromorphic p-valent, growth and distortion, radius of convexity, convex linear combinations. 
and $\Sigma_{p}^{C}(\alpha)$ defined above, were studied rather extensively by (among others) Aouf et al. ([4], [5]), Kulkarni et al. [8], Mogra ([6], [7]) and Srivastava et al. ([2], [3]).

A function $f$ given by (1.1) is said to be a member of the class $L(\alpha, \beta, \lambda, p)$ if it satisfies

$$
\left|\frac{z^{p+1} f^{\prime}(z)+p}{\alpha z^{p+1} f^{\prime}(z)+[p+(\lambda-\alpha)(p-\alpha)]}\right|<\beta
$$

where $0<\beta \leq 1,0<\lambda+p-\sqrt{(\lambda+p)^{2}-4 p(1+\lambda)}<\alpha<\lambda+p+\sqrt{(\lambda+p)^{2}-4 p(1+\lambda)}$ $\leq p,(\lambda+p)^{2}>4 p(1+\lambda)$ and $\lambda \geq \frac{p}{2}-2$ for all $z \in D$.

In this paper sharp results concerning coefficients, distortion theorem and the radius of convexity for the class $L(\alpha, \beta, \lambda, p)$ are determined. Finally we prove that the class is closed under convex linear combination.

\section{Coefficient Inequalities}

Our first result for functions $f \in L(\alpha, \beta, \lambda, p)$ is given as the following theorem.

Theorem 2.1. If $f \in \sum_{p}$ given by (1.1) satisfies

$$
\sum_{n=1}^{\infty}(p+n-1)(1+\alpha \beta)\left|a_{p+n-1}\right| \leq \beta[p(1-\alpha)+(\lambda-\alpha)(p-\alpha)]
$$

Then $f \in L(\alpha, \beta, \lambda, p)$, where $0<\beta \leq 1,0<\lambda+p-\sqrt{(\lambda+p)^{2}-4 p(1+\lambda)}<\alpha$ $<\lambda+p+\sqrt{(\lambda+p)^{2}-4 p(1+\lambda)} \leq p,(\lambda+p)^{2}>4 p(1+\lambda)$ and $\lambda \geq \frac{p}{2}-2$, for all $z \in D$.

Proof. Let us suppose that

$$
\sum_{n=1}^{\infty}(p+n-1)(1+\alpha \beta)\left|a_{p+n-1}\right| \leq \beta[p(1-\alpha)+(\lambda-\alpha)(p-\alpha)]
$$

for $f \in \sum_{p}$. Consider the expression

$$
M\left(f, f^{\prime}\right)=\left|z^{p+1} f^{\prime}(z)+p\right|<\beta\left|\alpha z^{p+1} f^{\prime}(z)+p+(\lambda-\alpha)(p-\alpha)\right| .
$$

Then for $0<|z|=r<1$ we have

$$
\begin{aligned}
M\left(f, f^{\prime}\right)= & \left|\sum_{n=1}^{\infty}(p+n-1) a_{p+n-1} z^{2 p+n-1}\right| \\
& -\beta\left|\sum_{n=1}^{\infty}(p+n-1) a_{p+n-1} z^{2 p+n-1}+p(1-\alpha)+(\lambda-\alpha)(p-\alpha)\right| .
\end{aligned}
$$




$$
\begin{aligned}
\frac{M\left(f, f^{\prime}\right)}{r^{p}} \leq & \left(\sum_{n=1}^{\infty}(p+n-1)\left|a_{p+n-1}\right| r^{p+n-1}\right) \\
& -\beta\left(-\sum_{n=1}^{\infty}(p+n-1)\left|a_{p+n-1}\right| r^{p+n-1}+\frac{p(1-\alpha)+(\lambda-\alpha)(p-\alpha)}{r^{p}}\right) \\
\leq & \sum_{n=1}^{\infty}(p+n-1)(1+\alpha \beta)\left|a_{p+n-1}\right| r^{p+n-1}-\beta[p(1-\alpha)+(\lambda-\alpha)(p-\alpha)] .
\end{aligned}
$$

The inequality above holds true for all $r(0<r<1)$. Thus by letting $r \rightarrow 1^{-}$into the inequality above, we obtain $M\left(f, f^{\prime}\right) \leq 0$. Hence it follows that (1.2) true, and so $f \in L(\alpha, \beta, \lambda, p)$.

The result is sharp for functions $f$ of the form

$$
f(z)=z^{-p}+\frac{\beta[p(1-\alpha)+(\lambda-\alpha)(p-\alpha)]}{(p+n-1)(1+\alpha \beta)} z^{p+n-1} \quad(n \geq 1) .
$$

Corollary 2.1. Let the function be defined by (1.1). If $f \in L(\alpha, \beta, \lambda, p)$, then

$$
\left|a_{p+n-1}\right| \leq \frac{\beta[p(1-\alpha)+(\lambda-\alpha)(p-\alpha)]}{(p+n-1)(1+\alpha \beta)}, \quad(n \geq 1) .
$$

The result is sharp for functions $f$ given by (2.4).

\section{Distortion Theorem}

A distortion property for functions in the class $f \in L(\alpha, \beta, \lambda, p)$, is given as follows:

Theorem 3.1. If the function $f$ given by (1.1) is in the class $f \in L(\alpha, \beta, \lambda, p)$, then for $0<|z|=r<1$ we have

$$
\frac{1}{r^{p}}-\frac{\beta[p(1-\alpha)+(\lambda-\alpha)(p-\alpha)]}{p(1+\alpha \beta)} r^{p} \leq|f(z)| \leq \frac{1}{r^{p}}+\frac{\beta[p(1-\alpha)+(\lambda-\alpha)(p-\alpha)]}{p(1+\alpha \beta)} r^{p}
$$

with equality for

$$
f(z)=\frac{1}{z^{p}}+\frac{\beta[p(1-\alpha)+(\lambda-\alpha)(p-\alpha)]}{p(1+\alpha \beta)} z^{p}, \quad z=(\text { ir }, r)
$$

and

$\frac{p}{r^{p+1}}-\frac{\beta[p(1-\alpha)+(\lambda-\alpha)(p-\alpha)]}{(1+\alpha \beta)} r^{p-1} \leq\left|f^{\prime}(z)\right| \leq \frac{p}{r^{p+1}}+\frac{\beta[p(1-\alpha)+(\lambda-\alpha)(p-\alpha)]}{(1+\alpha \beta)} r^{p-1}$. 
With equality for,

$$
f(z)=\frac{1}{z^{p}}+\frac{\beta[p(1-\alpha)+(\lambda-\alpha)(p-\alpha)]}{p(1+\alpha \beta)} z^{p}, \quad z=( \pm i r, \pm r)
$$

Proof. Since $f \in L(\alpha, \beta, \lambda, p)$, Theorem 2.1 yields the inequality

$$
\sum_{n=1}^{\infty}\left|a_{p+n-1}\right| \frac{\beta[p(1-\alpha)+(\lambda-\alpha)(p-\alpha)]}{(p+n-1)(1+\alpha \beta)} \leq 1
$$

Thus, for $0<|z|=r<1$, and making use of (2.3) we have

$$
\begin{aligned}
|f(z)| & =\left|\frac{1}{z^{p}}+\sum_{n=1}^{\infty} a_{p+n-1} z^{p+n-1}\right| \\
& \leq|z|^{-p}+\sum_{n=1}^{\infty} a_{p+n-1}|z|^{p+n-1} \\
& \left.\leq r^{-p}+\frac{\beta[p(1-\alpha)+(\lambda-\alpha)(p-\alpha)]}{(p+n-1)(1+\alpha \beta)}, \quad \text { (we substitute in }(3.5) \text { when } \mathrm{n}=1\right) \\
& \leq r^{-p}+\frac{\beta[p(1-\alpha)+(\lambda-\alpha)(p-\alpha)]}{p(1+\alpha \beta)} \\
& =\frac{1}{r^{p}}+\frac{\beta[p(1-\alpha)+(\lambda-\alpha)(p-\alpha)]}{p(1+\alpha \beta)} .
\end{aligned}
$$

And

$$
\begin{aligned}
|f(z)| & =\left|\frac{1}{z^{p}}+\sum_{n=1}^{\infty} a_{p+n-1} z^{p+n-1}\right| \\
& \geq|z|^{-p}-\sum_{n=1}^{\infty} a_{p+n-1}|z|^{p+n-1} \\
& \left.\geq r^{-p}-\frac{\beta[p(1-\alpha)+(\lambda-\alpha)(p-\alpha)]}{(p+n-1)(1+\alpha \beta)}, \quad \text { (we substitute in (3.5) when } \mathrm{n}=1\right) \\
& \geq r^{-p}-\frac{\beta[p(1-\alpha)+(\lambda-\alpha)(p-\alpha)]}{p(1+\alpha \beta)} \\
& =\frac{1}{r^{p}}-\frac{\beta[p(1-\alpha)+(\lambda-\alpha)(p-\alpha)]}{p(1+\alpha \beta)} .
\end{aligned}
$$

Also from Theorem 2.1, it follows that

$$
\sum_{n=1}^{\infty}(p+n-1)\left|a_{p+n-1}\right| \leq \frac{\beta[p(1-\alpha)+(\lambda-\alpha)(p-\alpha)]}{(1+\alpha \beta)} .
$$


Thus

$$
\begin{aligned}
\left|f^{\prime}(z)\right| & =\left|-p z^{-p-1}+\sum_{n=1}^{\infty}(p+n-1) a_{p+n-1} z^{p+n-2}\right| \\
& \leq p r^{-p-1}+\frac{\beta[p(1-\alpha)+(\lambda-\alpha)(p-\alpha)]}{(1+\alpha \beta)} r^{p+n-2}, \\
& \leq \frac{p}{r^{p+1}}+\frac{\beta[p(1-\alpha)+(\lambda-\alpha)(p-\alpha)]}{(1+\alpha \beta)} r^{p-1}
\end{aligned}
$$

and

$$
\begin{aligned}
\left|f^{\prime}(z)\right| & =\left|-p z^{-p-1}+\sum_{n=1}^{\infty}(p+n-1) a_{p+n-1} z^{p+n-2}\right| \\
& \geq p r^{-p-1}-\frac{\beta[p(1-\alpha)+(\lambda-\alpha)(p-\alpha)]}{(1+\alpha \beta)} r^{p+n-2}, \\
& \geq \frac{p}{r^{p+1}}-\frac{\beta[p(1-\alpha)+(\lambda-\alpha)(p-\alpha)]}{(1+\alpha \beta)} r^{p-1} .
\end{aligned}
$$

Hence completes the proof of Theorem 3.1.

\section{Radii of Starlikeness and Convexity}

The radii of starlikeness and convexity for the class $L(\alpha, \beta, \lambda, p)$, is given by the following theorem:

Theorem 4.1. If the function $f$ defined by (1.1) is in the class $L(\alpha, \beta, \lambda, p)$, then $f$ is starlike of order $\rho(0 \leq \rho<p)$, in the disk $|z|<r_{1}(\alpha, \beta, \lambda, p, \rho)$, where $r_{1}(\alpha, \beta, \lambda, p, \rho)$, is the largest value for which

$$
r_{1}=r_{1}(\alpha, \beta, \lambda, p, \rho)=\inf _{n \geq 1}\left(\frac{(p-\rho)[(p+n-1)(1+\alpha \beta)]}{(3 p+n-\rho-1) \beta[p(1-\alpha)+(\lambda-\alpha)(p-\alpha)]}\right)^{\frac{1}{2 p+n-1}} .
$$

The result is sharp for functions $f$ given by (2.4).

Proof. It suffices to show that

$$
\left|\frac{z f^{\prime}(z)}{f(z)}+p\right| \leq(p-\rho)
$$

For $|z| \leq r_{1}$, we have

$$
\left|\frac{z f^{\prime}(z)}{f(z)}+p\right|=\left|\frac{-p z^{-p}+\sum_{n=1}^{\infty}(p+n-1) a_{p+n-1} z^{p+n-1}}{z^{-p}+\sum_{n=1}^{\infty} a_{p+n-1} z^{p+n-1}}+p\right|
$$


which gives

$$
\begin{aligned}
\left|\frac{\sum_{n=1}^{\infty}(2 p+n-1) a_{p+n-1} z^{p+n-1}}{z^{-p}+\sum_{n=1}^{\infty} a_{p+n-1} z^{p+n-1}}\right| & =\left|\frac{\sum_{n=1}^{\infty}(2 p+n-1) a_{p+n-1} z^{2 p+n-1}}{1-\sum_{n=1}^{\infty} a_{p+n-1} z^{2 p+n-1}}\right| \\
& \leq \frac{\sum_{n=1}^{\infty} \frac{(2 p+n-1) \beta[p(1-p)+(\lambda-\alpha)(p-\alpha)])}{(p+n-1)(1+\alpha \beta)}|z|^{2 p+n-1}}{1-\sum_{n=1}^{\infty} \frac{(2 p+n-1) \beta[p(1-p)+(\lambda-\alpha)(p-\alpha)])}{(p+n-1)(1+\alpha \beta)}|z|^{2 p+n-1}} \\
& \leq(p-\rho) .
\end{aligned}
$$

The inequality above holds true if

$$
\sum_{n=1}^{\infty} \frac{(3 p+n-\rho-1) \beta[p(1-\alpha)+(\lambda-\alpha)(p-\alpha)]}{(p+n-1)(1+\alpha \beta)}|z|^{2 p+n-1} \leq(p-\rho),
$$

and it follows that

$$
|z| \leq\left(\frac{(p-\rho)[(p+n-1)(1+\alpha \beta)]}{(3 p+n-\rho-1) \beta[p(1-\alpha)+(\lambda-\alpha)(p-\alpha)]}\right)^{\frac{1}{2 p+n-1}}, \quad n \geq 1 .
$$

Then we have

$$
r_{1}(\alpha, \beta, \lambda, p, \rho)=\inf _{n \geq 1}\left(\frac{(p-\rho)[(p+n-1)(1+\alpha \beta)]}{(3 p+n-\rho-1) \beta[p(1-\alpha)+(\lambda-\alpha)(p-\alpha)]}\right)^{\frac{1}{2 p+n-1}}
$$

as required.

Theorem 4.2. If the function $f$ defined by (1.1) is in the class $L(\alpha, \beta, \lambda, p)$, then $f$ is convex of order $\rho(0 \leq \rho<p)$, in the disk $|z|<r_{2}(\alpha, \beta, \lambda, p, \rho)$, where $r_{2}(\alpha, \beta, \lambda, p, \rho)$, is the largest value for which

$$
r_{2}=r_{2}(\alpha, \beta, \lambda, p, \rho)=\inf _{n \geq 1}\left(\frac{p(p-\rho)(1+\alpha \beta)}{(3 p+n-\rho-1) \beta[p(1-\alpha)+(\lambda-\alpha)(p-\alpha)]}\right)^{\frac{1}{2 p+n-1}}
$$

The result is sharp for functions $f$ given by (2.4).

Proof. It suffices to show that

$$
\left|\frac{z f^{\prime \prime}(z)}{f^{\prime}(z)}+(1+p)\right| \leq(p-\rho)
$$


For $|z| \leq r_{2}$, we have

$$
\begin{aligned}
& \left|\frac{z f^{\prime \prime}(z)}{f^{\prime}(z)}+(1+p)\right| \\
= & \left|\frac{\left(p^{2}+p\right) z^{-p-1}+\sum_{n=1}^{\infty}(p+n-1)(p+n-2) a_{p+n-1} z^{p+n-2}}{-p z^{-p-1}+\sum_{n=1}^{\infty}(p+n-1) a_{p+n-1} z^{p+n-2}}+(p+1)\right| \\
= & \left|\frac{\sum_{n=1}^{\infty}(2 p+n-1)(p+n-1) a_{p+n-1} z^{2 p+n-1}}{-p+\sum_{n=1}^{\infty}(p+n-1) a_{p+n-1} z^{2 p+n-1}}\right| \\
\leq & \frac{\sum_{n=1}^{\infty}(2 p+n-1)(p+n-1) \frac{\beta[p(1-p)+(\lambda-\alpha)(p-\alpha)])}{(p+n-1)(1+\alpha \beta)}|z|^{2 p+n-1}}{p-\sum_{n=1}^{\infty}(p+n-1) \frac{\beta[p(1-p)+(\lambda-\alpha)(p-\alpha)])}{(p+n-1)(1+\alpha \beta)}|z|^{2 p+n-1}} \leq(p-\rho) .
\end{aligned}
$$

The inequality above holds true if

$$
\sum_{n=1}^{\infty} \frac{[(3 p+n-\rho-1)] \beta[p(1-\alpha)+(\lambda-\alpha)(p-\alpha)]}{(1+\alpha \beta)}|z|^{2 p+n-1} \leq p(p-\rho),
$$

and it follows that

$$
|z| \leq\left(\frac{p(p-\rho)(1+\alpha \beta)}{[(3 p+n-\rho-1)] \beta[p(1-\alpha)+(\lambda-\alpha)(p-\alpha)]}\right)^{\frac{1}{2 p+n-1}}, \quad n \geq 1 .
$$

Then we have

$$
r_{2}(\alpha, \beta, \lambda, p, \rho)=\inf _{n \geq 1}\left(\frac{p(p-\rho)(1+\alpha \beta)}{(3 p+n-\rho-1) \beta[p(1-\alpha)+(\lambda-\alpha)(p-\alpha)]}\right)^{\frac{1}{2 p+n-1}}
$$

as required.

\section{Convex Linear Combination}

Our next result involves a linear combination of function $f$ of the type (2.4).

Theorem 5.1 Let

$$
f_{p}(z)=z^{-p}
$$

and

$$
f_{p+n-1}(z)=z^{-p}+\frac{\beta[p(1-\alpha)+(\lambda-\alpha)(p-\alpha)]}{(p+n-1)(1+\alpha \beta)} z^{p+n-1}, \quad(n \geq 1) .
$$


Then $f \in L(\alpha, \beta, \lambda, p)$ if and only if it can be expressed in the form

$$
\begin{gathered}
f(z)=\sum_{n=1}^{\infty} \lambda_{p+n-1} f_{p+n-1}(z) \\
\text { where } \quad \lambda_{p+n-1} \geq 0 \quad \text { and } \quad \sum_{n=1}^{\infty} \lambda_{p+n-1}=1 .
\end{gathered}
$$

Proof. From (5.12), (5.13) and (5.14), it is easily seen that

$$
\begin{aligned}
f(z) & =\sum_{n=1}^{\infty} \lambda_{p+n-1} f_{p+n-1}(z), \\
& =\sum_{n=1}^{\infty} \lambda_{p+n-1}\left(z^{-p}+\frac{\beta[p(1-\alpha)+(\lambda-\alpha)(p-\alpha)]}{(p+n-1)(1+\alpha \beta)} z^{p+n-1}\right), \\
& =z^{-p}+\sum_{n=1}^{\infty} \frac{\beta[p(1-\alpha)+(\lambda-\alpha)(p-\alpha)] \lambda_{p+n-1}}{(p+n-1)(1+\alpha \beta)} z^{p+n-1} .
\end{aligned}
$$

Then it follows that

$$
\sum_{n=1}^{\infty} \frac{(p+n-1)(1+\alpha \beta)}{\beta[p(1-\alpha)+(\lambda-\alpha)(p-\alpha)]} \frac{\beta[p(1-\alpha)+(\lambda-\alpha)(p-\alpha)] \lambda_{p+n-1}}{(p+n-1)(1+\alpha \beta)}
$$

and

$$
\sum_{n=2}^{\infty} \lambda_{p+n-1}=1-\lambda_{p} \leq 1
$$

So, by Theorem 2.1 we have $f \in L(\alpha, \beta, \lambda, p)$. Conversely, let us suppose that $f \in$ $L(\alpha, \beta, \lambda, p)$. Then

$$
a_{p+n-1} \leq \frac{\beta[p(1-\alpha)+(\lambda-\alpha)(p-\alpha)]}{(p+n-1)(1+\alpha \beta)}, \quad(n \geq 1) .
$$

Setting

$$
\lambda_{p+n-1}=\frac{(p+n-1)(1+\alpha \beta)}{\beta[p(1-\alpha)+(\lambda-\alpha)(p-\alpha)]} a_{p+n-1} \quad(n \geq 1)
$$

and $\lambda_{p}=1-\sum_{n=2}^{\infty} \lambda_{p+n-1}$. It follows that

$$
f(z)=\sum_{n=1}^{\infty} \lambda_{p+n-1} f_{p+n-1}(z)
$$

and the proof of the theorem is complete. Finally we prove

Theorem 5.2. The class $L(\alpha, \beta, \lambda, p)$ is closed under convex linear combinations. 
Proof. Suppose that the functions $f_{1}$ and $f_{2}$ defined by,

$$
f_{i}(z)=z^{-p}+\sum_{n=1}^{\infty} a_{p+n-1, i} z^{p+n-1} \quad(i=1,2 ; z \in D)
$$

are in the class $L(\alpha, \beta, \lambda, p)$.

Setting $h(z)=\mu f_{1}(z)+(1-\mu) f_{2}(z)$ we want to show that $f \in L(\alpha, \beta, \lambda, p)$.

For $0 \leq \mu \leq 1$, we can write

$$
\begin{aligned}
h(z) & =z^{-p}+\mu \sum_{n=1}^{\infty} a_{p+n-1,1}+(1-\mu) \sum_{n=1}^{\infty} a_{p+n-1,2} z^{p+n-1}, \\
& =z^{-p}+\sum_{n=1}^{\infty}\left\{\mu a_{p+n-1,1}+(1-\mu) a_{p+n-1,2}\right\} z^{p+n-1}, \quad(i=1,2 ; z \in D) .
\end{aligned}
$$

In view of Theorem 2.1, we have

$$
\begin{aligned}
& \sum_{n=1}^{\infty}[(p+n-1)(1+\alpha \beta)]\left\{\mu a_{p+n-1,1}+(1-\mu) a_{p+n-1,2}\right\} \\
= & \mu \sum_{n=1}^{\infty}[(p+n-1)(1+\alpha \beta)] a_{p+n-1,1}+(1-\mu) \sum_{n=1}^{\infty}[(p+n-1)(1+\alpha \beta)] a_{p+n-1,2} \\
\leq & \mu \beta[p(1-\alpha)+(\lambda-\alpha)(p-\alpha)]+(1-\mu) \beta[p(1-\alpha)+(\lambda-\alpha)(p-\alpha)] \\
= & \beta[p(1-\alpha)+(\lambda-\alpha)(p-\alpha)]
\end{aligned}
$$

which shows that $f \in L(\alpha, \beta, \lambda, p)$. Hence the theorem.

\section{Acknowledgement}

The work presented here was supported by IRPA 09-02-02-10029 EAR, Malaysia.

\section{References}

[1] P. L. Duren, Univalent functions, In Grundlehren der Mathematischen Wissenschaften, 259, Springer-Verlag, New York, 1983.

[2] H. M. Srivastava, H. M. Hossen and M. K. Aouf., A unified presentation of some classes meromorphically multivalent functions, Comp. Math. with App. 38(1999), 63-70.

[3] H. M. Srivastava and M. K. Aouf, Some applications of fractional calculus operators to certain subclasses of prestarlike functions with negative coefficients, Comp. Math. with App. 30,(1995), 53-61.

[4] M. K. Aouf, On a class meromorphic multivalent functions with positive coefficients, Math. Japan. 35, (1990), 603-608.

[5] M. K. Aouf, A generalization of meromorphic multivalent functions with positive coefficients, Math. Japan. 35, (1990), 609-614. 
[6] M. L. Mogra, Meromorphic multivalent functions with positive coefficients I, Math. Japan. 35, (1990), 1-11.

[7] M. L. Mogra, Meromorphic multivalent functions with positive coefficients II, Math. Japan. 35, (1990), 1089-1098

[8] S. R. Kulkarni, U. H. Naik and H. M. Srivastava, A certain class of meromorphically pvalent, quasi-convex functions, Pan Amer. Math. J. 8 (1998), 57-64.

School of Mathematical Sciences, Faculty of Science and Technology, Universiti Kebangsaan Malaysia, Bangi 43600 Selangor D.E., Malaysia.

E-mail: maslina@pkrisc.cc.ukm.my 